\title{
ORIGINAL ARTICLE \\ Prebiotic and synbiotic effects on rats fed malted barley with selected bacteria strains
}

\author{
Yadong Zhong* and Margareta Nyman \\ Applied Nutrition and Food Chemistry, Department of Food Technology, Engineering and Nutrition, Kemicentrum, \\ Lund University, Lund, Sweden
}

Abstract

Background: Butyric acid, one of the key products formed when $\beta$-glucans are degraded by the microbiota in the colon, has been proposed to be important for colonic health. Glutamine bound to the fibre may have similar effects once it has been liberated from the fibre in the colon. Both $\beta$-glucans and glutamine are found in high amounts in malted barley. Lactobacillus rhamnosus together with malt has been shown to increase the formation of butyric acid further in rats.

Objective: To investigate whether Lactobacillus rhamnosus 271, Lactobacillus paracasei 87002, Lactobacillus plantarum HEAL 9 and 19, and Bifidobacterium infantis CURE 21 affect the levels of short-chain fatty acids and glutamine in caecum and portal blood of rats fed barley malt.

Design: The experimental diets were fed for 12 days. The daily dose of the probiotic strain was $1 \times 10^{9}$ colony forming units and the intake of fibre $0.82 \mathrm{~g} /$ day.

Results: The malt mostly contained insoluble fibre polymers (93\%), consisting of glucose and xylose (38-41 $\mathrm{g} / \mathrm{kg}$ ) and some arabinose $(21 \mathrm{~g} / \mathrm{kg})$. The fibre polysaccharides were quite resistant to fermentation in the rats, regardless of whether or not probiotics were added (25-30\% were fermented). Caecal and portal levels of acetic acid decreased in the rats after the addition of L. plantarum HEAL 9 and L. rhamnosus 271, and also the levels of butyric acid. Viable counts of Lactobacillus, Bifidobacterium and Enterobacteriaceae were unaffected, while the caecal composition of Lactobacilli was influenced by the type of strain administrated. Portal levels of glutamine were unchanged, but glycine levels increased with L. plantarum HEAL 9 and 19 and phenylalanine with L. rhamnosus 271.

Conclusions: Although the probiotic strains survived and reached the caecum, except B. infantis CURE 21, there were no effects on viable counts or in the fermentation of different fibre components, but the formation of some bacterial metabolites decreased. This may be due to the high proportion of insoluble fibres in the malt.

Keywords: dietary fibre; probiotics; short-chain fatty acids; amino acids; microbiota

Responsible Editor: Asim Duttaroy, Oslo University, Norway.

Received: 8 May 20I4; Revised: 2 September 20।4; Accepted: 5 September 20I4; Published: 6 October 2014

$\circlearrowleft$ hort-chain fatty acids (SCFAs), preferably butyric acid and propionic acid, formed by colonic fermentation of dietary fibre have been associated with a number of health-promoting effects. Butyric acid is the main substrate for the colonocytes and may play an important role in the prevention and treatment of colonic diseases such as ulcerative colitis and colon cancer $(1,2)$. Butyric acid has also been reported to increase colonic blood flow and oxygen uptake (3), and to stimulate mucosal proliferation $(4,5)$. On the other hand, butyric acid may also act as an inhibitor of inflammation in the colon, via the inhibition of $N F-\kappa B$ activation and histone deacetylation (6). This inhibiting effect of butyric acid has also been seen on systemic inflammation (7).
Another interesting SCFA is propionic acid, which may inhibit the synthesis of cholesterol from acetic acid and lower plasma glucose level, thereby playing a role in modulating lipid and glucose metabolism $(8,9)$.

The profile of SCFAs in the colon has been shown to rely on the type of dietary fibre, the degree of polymerisation of the dietary fibre, the type of linkages between the carbohydrate monomers, and on solubility and monomeric composition (10). While pectin (polymers containing high amounts of uronic acids) gives high amounts of acetic acid, guar gum (galactomannan) yields high proportions of propionic acid and $\beta$-glucan high amounts of butyric acid (10-12). Furthermore, fructan of low molecular weight (fructo-oligosaccharides) gives high amounts 
of butyric acid, in contrast to high-molecular-weight fructan (inulin) (10-12), and inulin with low solubility tends to shift the fermentation site from the caecum to the distal colon in rats (10). Thus, the physico-chemical properties of the fibre are of great importance for the amount and pattern of SCFAs formed.

Apart from the type of carbohydrate, SCFA formation also depends on the composition of the colonic microbiota. One way to improve the profile of the colonic microbiota is by adding probiotics. In recent years, there has been considerable interest in studying supplementation of probiotics with dietary fibre, as a possible way to optimise SCFA formation and enhance the nutritional effect of dietary fibre. For example, a shift in the formation of SCFAs from the caecum to the distal colon was observed in rats fed pectin with Lactobacillus UCC500, and Bifidobacterium supplementation (Bb-12) increased the levels of SCFAs throughout the colon of rats fed pectin (13).

Barley malt, which is traditionally used in the brewing industry, is the product of the germination of barley. During germination, the non-starch polysaccharides (NSPs) in the cell wall of barley, constituting mainly arabinoxylans and $\beta$-glucans, are solubilised and partly degraded into smaller molecules (14). These changes probably make NSPs more sensitive to the bacterial fermentation in the human colon. In a previous study, we have shown that feeding rats with barley malt gave rise to higher levels of propionic and butyric acids than the starting material (whole grain barley), and that supplementation with a probiotic strain, Lactobacillus rhamnosus 271 (Lr 271), increased the formation further (15). Increased levels of theses acids in the circulation system may have metabolic effects and influence inflammatory parameters. Furthermore, barley and germinated barley contain large quantities of glutamine $(16,17)$, which after butyric acid, is the most important substrate for colonic mucosa. Glutamine is easily degraded by the low $\mathrm{pH}$ in the stomach, but some of the glutamine is protected by the dietary fibre during digestion in the stomach (17), and therefore reaches the colon. This is also a proposed explanation of why germinated barley prolongs remission in subjects with ulcerative colitis (18). Greater formation of butyric acid may reduce the epithelial utilisation of glutamine, thereby increasing the levels of circulating glutamine affecting the immune function.

The aim of the present study was to evaluate the promising synbiotic effect of dietary fibre and some probiotic strains. For this purpose, barley malt, and Lactobacillus paracasei 87002 (Paracasei), Lactobacillus plantarum HEAL 9 (HEAL 9) and 19 (HEAL 19) and Bifidobacterium infantis CURE 21 (CURE 21) were used. To verify previous effects, $\mathrm{Lr} 271$ was also included in diets given to one group of rats. The rat model used has been shown to correlate well to human experiments (19). The degree of dietary fibre fermentation, formation of SCFAs in the hindgut, levels of SCFAs and amino acids in the portal blood, and the composition of Lactobacillus in caecum were investigated. Paracasei has been shown to have strong antagonistic properties, HEAL 9 and 19 were chosen because of their ability to adhere to human epithelial cells and their capacity to break down tannins and CURE 21 can produce glutamine (20-22).

\section{Materials and methods}

\section{Materials}

Malt was obtained free of charge by Carlsberg AB (Falkenberg, Sweden), and milled to a particle size less than $0.5 \mathrm{~mm}$ before inclusion in the diets. Five lyophilised probiotics, Paracasei, HEAL 9, HEAL 19, CURE 21 and Lr 271 were obtained from Probi AB (Lund, Sweden).

\section{Diets}

The six diets used in the study contained a basal diet mixture $(329 \mathrm{~g} / \mathrm{kg}$, dry weight) and were prepared according to Henningsson et al. (23). Malt was added at a level of $68 \mathrm{~g}$ dietary fibre per $\mathrm{kg}$ dry feed (corresponding to $481 \mathrm{~g}$ malt per $\mathrm{kg}$ feed, dry weight basis, dwb), and wheat starch $(189.7 \mathrm{~g} / \mathrm{kg}, \mathrm{dwb})$ was used to adjust the dry matter weight. The starch used makes no contribution to the hindgut formation of SCFAs due to complete digestion in the upper gastrointestinal tract (24). The malt diet without probiotics is referred to as the control diet, and the other five diets containing one of the five probiotic strains are referred to as test diets. The daily amount of probiotics was $1 \times 10^{9}$ colony forming units (CFU) for each rat, and was added at feeding time.

\section{Animal study design}

Male Wistar rats $(n=42)$ obtained from Taconic (Ejby, Denmark) were used in the study. The rats were randomly divided into six groups and housed individually in metabolic cages in a room maintained at $22^{\circ} \mathrm{C}$ and $60 \%$ relative humidity in a 12 -h light and night circle. After 1 week of acclimatisation to the environment, the rats $(131.5 \pm 1.1 \mathrm{~g})$ were allowed to adapt to the diet for 7 days before a 5-day long experimental period. Food intake was restricted to $12 \mathrm{~g}(\mathrm{dwb})$ per day, and water was given ad libitum. During the experimental period, faeces and feed residues were collected. Faecal material was freeze-dried and milled before analysis of the content of dietary fibre monomers. At the end of the experiment, the rats were anaesthetised by injecting Hypnorm-Dormicum in sterile water (1:1:2) at a dose of $0.15 \mathrm{ml}$ per $100 \mathrm{~g}$ body weight. Portal blood was taken for analysis of SCFAs, amino acids and ammonia. Caecal content and proximal and distal colon content were collected for analysis of SCFAs. Caecal $\mathrm{pH}$ and the weight of caecal content and tissue were measured directly. The animal experiment was 
approved by the Ethics Committee for Animal Studies at Lund University (M 30-09).

\section{Analyses \\ Dietary fibre}

The soluble and insoluble dietary fibres in malt were determined with an enzymatic, gravimetric method (25). The Uppsala Method was used to analyse the monomeric composition of the dietary fibre residues and also dietary fibre in faeces (26), but without the step involving isolation of the dietary fibre. In this method, the dietary fibre residues are first hydrolysed and filtered. The filtrate is then used to analyse neutral sugars by a gas chromatographic (GC) method, as their alditol acetates, and uronic acids by a spectrophotometric method. The content of Klason lignin in the samples was determined as the residue after filtration and combustion of the sample at $550^{\circ} \mathrm{C}$ for $1 \mathrm{~h}$.

\section{SCFAs in caecum, colon and serum}

A GC method was applied for the analysis of SCFAs in caecal and colonic contents (27) and in serum (28). The caecal and colonic contents were homogenised (IKA ${ }^{\circledR}$ WERKE, Staufen, Germany) and centrifuged with $0.25 \mathrm{M}$ $\mathrm{HCl}$ to protonise the SCFAs. SCFAs in serum were enriched by a hollow fibre supported liquid membrane extraction technique. Two-ethylbutyric acid (Sigma Chemical Company) was added in each sample as internal standard. After preparation, the samples were injected into a fused-silica capillary column (DB-FFAP 125-3237; J\&W Scientific, Agilent Technologies Inc., Folsom, CA, USA). GC ChemStation software (Agilent Technologies Inc., Wilmington, DE, USA) was used for the evaluation of the results.

\section{Amino acids in plasma}

Amino acids in plasma were quantified as free amino acids with an amino acid analyser (Biochrom 30, Biochrom Ltd, Cambridge, UK) based on ion-exchange chromatography. A precipitation step was used to purify the plasma (29). The EZChrom Elite software package (Scientific Software Inc., Pleasanton, CA, USA) was used to evaluate the results.

\section{Caecal microbiota}

Viable counts of Bifidobacterium, Lactobacillus and Enterobacteriaceae in caecal samples were performed by anaerobical incubation, as described elsewhere (30). Colonies of Bifidobacterium were randomly selected and verified by microscopy. Four Lactobacillus colonies obtained from each cultured sample were randomly picked and re-cultured on Rogosa agar as the quantitatively dominant lactobacilli in the caecum of rats. The randomly amplified polymorphic DNA method (RAPD) was used to identify the given probiotic strain from the dominant Lactobacilli, followed by agarose gel electrophoresis (31).
The unknown Lactobacilli were sent for sequencing to identify them at species level (GATC Biotech, Solna, Sweden).

\section{Statistics}

One rat fed malt + CURE 21 suffered a weight loss $(6.4 \mathrm{~g})$ during the experiment and showed very low concentrations of SCFAs both in serum and caecal content. The data from this rat were therefore excluded from the statistical analysis as this animal was considered an outlier.

The caecal pool was calculated as the level of each SCFA obtained in the analysis multiplied by the amount of caecal content, which was extrapolated to the complete intake of dietary fibre (4.08 g). Body weight gain during the experimental period was calculated as $\mathrm{g} / \mathrm{g}$ feed intake. Bulking capacity was calculated as faecal dry weight in $\mathrm{g} / \mathrm{g}$ fibre ingested.

The results are presented as means and their standard error means (SEM). Effects of probiotics were evaluated by one-way ANOVA followed by Dunnett's procedure, where the test diets were compared $(P<0.05)$ with the control diet. Box-Cox transformation was carried out when data was not normally distributed. Minitab version 14 was used for statistical analysis.

\section{Results}

Dietary fibre content, composition and hindgut degradation The content of dietary fibre polysaccharides in malt was $110 \mathrm{~g} / \mathrm{kg}(\mathrm{dwb})$, of which only a small part was soluble (7\%) (Table 1). The main components of the dietary fibre polysaccharides were xylose (37\%), glucose $(34 \%)$ and arabinose $(19 \%)$. Considerable amounts of uronic acids $(5 \%)$, galactose and mannose $(5 \%)$ were also detected. The amount of Klason lignin was $25 \mathrm{~g} / \mathrm{kg}$ (dwb).

Dietary fibre polysaccharides in malt were rather resistant to hindgut degradation in rats and only $30 \%$ of the ingested fibre was fermented. Among the dietary fibre components, glucose was most resistant to fermentation, and only $14 \%$ was utilised by the hindgut microbiota. The other main components, xylose and arabinose were utilised to 32 and $52 \%$, respectively, by the hindgut microbiota. The minor components were fermented to similar degrees: $37-58 \%$. None of the probiotic strains affected the fermentability of the dietary fibre to any greater extent. The only significance seen was the higher degree of fermentation with malt and Paracasei compared with malt and CURE 21 or Lr 271 when suing another statistical test, that is, student's $t$-test, than one-way ANOVA.

\section{Caecal content, tissue weight and $\mathrm{pH}$}

The rats appeared to be healthy throughout the study and ate most of the food provided during the experimental period (11.2-11.8 g/day), which corresponded to $93-98 \%$ 
Table 1. Dietary fibre content $(\mathrm{g} / \mathrm{kg})$, soluble fraction ( $\%$ of total dietary fibre) and faecal excretion $(\%$ of ingested fibre) in rats fed malt and malt together with Paracasei, HEAL 9, HEAL 19, CURE 21 or Lr 271

\begin{tabular}{|c|c|c|c|c|c|c|c|c|c|}
\hline & \multicolumn{2}{|c|}{ Dietary fibre } & \multicolumn{7}{|c|}{ Faecal excretion of dietary fibre } \\
\hline & Total & Soluble fraction & Malt & + Paracasei & + HEAL 9 & + Heal 19 & +CURE 21 & $+\operatorname{Lr} 27 I$ & $P$ \\
\hline Rhamnose & ND & ND & - & - & - & - & - & - & - \\
\hline Arabinose & 21 & 11 & $48 \pm 2$ & $49 \pm 2$ & $51 \pm 2$ & $52 \pm 2$ & $53 \pm 2$ & $53 \pm 1$ & 0.263 \\
\hline Xylose & 41 & 6 & $68 \pm 3$ & $69 \pm 2$ & $70 \pm 2$ & $71 \pm 3$ & $72 \pm 2$ & $72 \pm 1$ & 0.607 \\
\hline Mannose & 2 & 15 & $42 \pm 2$ & $37 \pm 4$ & $42 \pm 2$ & $43 \pm 2$ & $43 \pm 2$ & $46 \pm 2$ & 0.147 \\
\hline Galactose & 3 & 25 & $63 \pm 4$ & $60 \pm 3$ & $64 \pm 2$ & $63 \pm 3$ & $73 \pm 4$ & $67 \pm 4$ & 0.159 \\
\hline Glucose & 38 & 6 & $86 \pm 3$ & $86 \pm 4$ & $87 \pm 3$ & $87 \pm 3$ & $90 \pm 3$ & $93 \pm 3$ & 0.546 \\
\hline Uronic acids & 5 & 16 & $63 \pm 1$ & $61 \pm 3$ & $68 \pm 3$ & $68 \pm 3$ & $70 \pm 2$ & $70 \pm 2$ & 0.041 \\
\hline Dietary fibre polysaccharides & 110 & 8 & $70 \pm 2$ & $70 \pm 3$ & $72 \pm 2$ & $72 \pm 3$ & $74 \pm 2$ & $75 \pm 2$ & 0.534 \\
\hline Klason lignin & 25 & 0 & - & - & - & - & - & - & - \\
\hline Total dietary fibre & 135 & 7 & - & - & - & - & - & - & - \\
\hline
\end{tabular}

Results are given as means \pm SEM, $n=5$ for Malt + Paracasei, $n=6$ for Malt + CURE $2 I$ and $n=7$ for all other groups; ND, not detected at a detection limit of $0.5 \mathrm{~g} / \mathrm{kg}$.

of the feed intake (Table 2). All the rats included in the analysis gained weight, and no significances were detected between the groups.

Caecal content and tissue weight were similar in rats fed different diets $(2.2 \pm 0.1$ and $0.53 \pm 0.01 \mathrm{~g}$, respectively). The caecal $\mathrm{pH}$ was between 6.5 and 6.9 , with no differences between groups. No significant difference was observed in the bulking capacity between groups (1.38-1.49 g/g fibre).

\section{SCFAs in the hindgut}

The main SCFA formed in the caecum of all rats was acetic acid $(104-131 \mu \mathrm{mol})$, followed by butyric acid (22-36 $\mu \mathrm{mol})$ and propionic acid $(16-21 \mu \mathrm{mol})($ data not shown), which corresponded to $67-70 \%, 14-18 \%$, and $10-12 \%$ of the total amount of SCFAs formed, respectively.

The caecal levels of total SCFAs were lower in rats fed malt together with HEAL $9(P<0.01)$, HEAL 19 $(P<0.01)$ and $\operatorname{Lr} 271 \quad(P<0.05)$ than without these strains, mainly due to a decrease in the levels of acetic acid $(P<0.01,0.01$ and 0.05 , respectively), but also due to decreased levels of butyric acid $(P<0.01$ for HEAL 9 and $P<0.05$ for HEAL 19). The probiotic groups had lower levels of SCFAs in the distal colon than the group fed diets containing no probiotics, although this was not significant (Table 3).

\section{SCFAs in the portal blood}

Acetic acid $(820-1,090 \mu \mathrm{mol} / \mathrm{l})$, propionic acid $(45-77$ $\mu \mathrm{mol} / \mathrm{l})$ and butyric acid $(55-130 \mu \mathrm{mol} / \mathrm{l})$ were the major SCFAs presented in portal serum (Table 3). Considerable amounts of isobutyric acid $(12-14 \mu \mathrm{mol} / \mathrm{l})$, isovaleric acid $(13-15 \mu \mathrm{mol} / \mathrm{l})$ and valeric acid $(4-10 \mu \mathrm{mol} / \mathrm{l})$ were also detected (data not shown).
Levels of total SCFAs in the portal serum of rats were lower with malt together with HEAL 9 and Lr 271 than in rats fed only malt $(P<0.05)$, which was mainly due to the lower levels of acetic acid $(P<0.05)$. There was also a lower level of butyric acid in the portal serum of rats together with malt and HEAL 9 than with malt only $(P<0.05)$ (Table 3$)$.

\section{Amino acids in portal plasma}

The main amino acids in the portal plasma were glutamine $(16 \%)$, alanine $(14 \%)$, lysine $(13 \%)$ and glycine $(10 \%)$. Nineteen $\alpha$-amino acids and ammonia were analysed in the portal blood, but only ammonia, glutamine and those showing significant differences between rats fed malt alone and malt with probiotics are shown in Table 4, that is, asparagine, proline, phenylalanine and glycine. Plasma levels of glycine from rats given malt together with HEAL $9(P<0.001)$ or $19(P<0.05)$ were higher than with only malt, while the levels of asparagine $(P<0.05)$ and proline $(P<0.01)$ were higher when CURE 21 was added to the

Table 2. Feed intake, weight gain, caecal content, caecal tissue weight, caecal $\mathrm{pH}$ and bulking capacity in rats fed malt and malt together with Paracasei, HEAL 9, HEAL 19, CURE 21 or Lr 271

\begin{tabular}{lc}
\hline & Malt \pm probiotics \\
\hline Feed intake $(\mathrm{g} /$ day $)$ & $11.2-11.8$ \\
Weight gain $(\mathrm{g} / \mathrm{g}$ feed $)$ & $0.16-0.23$ \\
Caecal content $(\mathrm{g})$ & $2.1-2.4$ \\
Caecal tissue $(\mathrm{g})$ & 0.5 \\
Caecal $\mathrm{pH}$ & $6.5-6.8$ \\
Bulking capacity $(\mathrm{g} / \mathrm{g}$ fibre) & $1.4-1.5$ \\
\hline
\end{tabular}

All feed weights are given on a dry weight basis. Results are given as means \pm SEM, $n=6$ for malt + CURE $2 I$ and $n=7$ for all other groups. 
Table 3. Levels of SCFAs in the caecum $(\mu \mathrm{mol} / \mathrm{g})$, distal colon $(\mu \mathrm{mol} / \mathrm{g})$ and portal serum $(\mu \mathrm{mol} / \mathrm{l})$ of rats fed malt and malt together with Paracasei, HEAL 9, HEAL 19, CURE 21 or Lr 271

\begin{tabular}{|c|c|c|c|c|c|c|c|}
\hline & Malt & + Paracasei & + HEAL 9 & + HEAL 19 & + CURE 21 & $+\operatorname{Lr} 27 I$ & $P$ \\
\hline \multicolumn{8}{|l|}{ Caecum } \\
\hline Acetic & $60 \pm 5$ & $49 \pm 3$ & $42 \pm 3^{* *}$ & $42 \pm 2^{* *}$ & $55 \pm 3$ & $44 \pm 3^{*}$ & 0.003 \\
\hline Propionic & $9 \pm 1$ & $7 \pm 1$ & $7 \pm 1$ & $7 \pm 1$ & $9 \pm 0$ & $7 \pm 1$ & 0.059 \\
\hline Butyric & $14 \pm 2$ & $12 \pm 1$ & $8 \pm I^{* *}$ & $9 \pm 1 *$ & $15 \pm 2$ & $10 \pm 1$ & 0.003 \\
\hline Minor & $4 \pm 0$ & $3 \pm 0$ & $3 \pm 0$ & $3 \pm 0$ & $3 \pm 0$ & $3 \pm 0$ & 0.269 \\
\hline Total & $86 \pm 7$ & $7 I \pm 5$ & $60 \pm 5^{* *}$ & $61 \pm 5^{* *}$ & $82 \pm 5$ & $64 \pm 4^{*}$ & 0.002 \\
\hline \multicolumn{8}{|l|}{ Distal colon } \\
\hline Acetic & $39 \pm 9$ & $27 \pm 1$ & $26 \pm 2$ & $28 \pm 2$ & $35 \pm 5$ & $28 \pm 3$ & 0.164 \\
\hline Propionic & $8 \pm 2$ & $6 \pm 0$ & $6 \pm 1$ & $6 \pm 0$ & $7 \pm 1$ & $6 \pm 0$ & 0.080 \\
\hline Butyric & $11 \pm 3$ & $8 \pm 1$ & $7 \pm 1$ & $9 \pm 1$ & $10 \pm 1$ & $8 \pm 1$ & 0.077 \\
\hline Minor & $5 \pm 2$ & $3 \pm 1$ & $4 \pm I$ & $4 \pm 2$ & $5 \pm 2$ & $4 \pm 1$ & 0.506 \\
\hline Total & $64 \pm 13$ & $44 \pm 2$ & $42 \pm 5$ & $48 \pm 4$ & $58 \pm 8$ & $45 \pm 4$ & 0.118 \\
\hline \multicolumn{8}{|l|}{ Portal serum } \\
\hline Acetic & $1,095 \pm 70$ & $897 \pm 50$ & $837 \pm 38^{*}$ & $884 \pm 54$ & $983 \pm 111$ & $818 \pm 56^{*}$ & 0.035 \\
\hline Propionic & $69 \pm 10$ & $61 \pm 6.7$ & $45 \pm 6$ & $55 \pm 9$ & $77 \pm 16$ & $48 \pm 6$ & 0.103 \\
\hline Butyric & $103 \pm 22$ & $88 \pm 12$ & $55 \pm 6^{*}$ & $69 \pm 11$ & $127 \pm 38$ & $67 \pm 8$ & 0.027 \\
\hline Minor & $27 \pm 3$ & $27 \pm 2$ & $29 \pm 3$ & $3 I \pm 3$ & $32 \pm 5$ & $26 \pm 1$ & 0.806 \\
\hline Total & $1,356 \pm 103$ & $1,139 \pm 67$ & $1,040 \pm 49$ & $1,115 \pm 63$ & $1,296 \pm 117$ & $\mathrm{I}, 024 \pm 68^{*}$ & 0.056 \\
\hline
\end{tabular}

Results are given as means \pm SEM, $n=6$ for malt + CURE $2 I$ and $n=7$ for all other groups. Mean values were significantly different from those for rats fed with malt: $* P<0.05, * * P<0.01$.

malt diet than without this strain. Lr 271 contributed to higher levels of proline $(P<0.05)$ and phenylalanine $(P<0.01)$ in the rats. None of the probiotic strains affected the glutamine levels (Table 4).

\section{Caecal microbiota}

The viable counts of Lactobacillus, Bifidobacterium and Enterobacteriaceae in the caecal content were not affected by any of the probiotic strains added (Fig. 1), although all the strains given to the rats, according to the supplier, survived and reached the caecum. In rats fed Paracasei, HEAL 9 or 19 or Lr 271, the given probiotic strain was also the dominant Lactobacillus in the caecum content (Table 5). Apart from the administered probiotic strains, two other Lactobacilli were isolated and found in all groups, an exception was in rats fed HEAL 19 where only one of these strains was found. One of the strains belonged to species of Lactobacillus animalis and the other belonged to species of Lactobacillus reuteri. CURE 21 was not detected in the caecum of rats, which may be due to that the strain did not survive in the passage through the hindgut or to the limited culture technique.

\section{Discussion}

No increase in caecal or portal levels of propionic or butyric acid were seen with any of the probiotic strains, and the results from a previous study with an increased formation of propionic and butyric acids with barley malt

Table 4. Plasma levels of amino acids $(\mu \mathrm{mol} / \mathrm{l})$ in portal blood of rats fed malt and malt together with Paracasei, HEAL9, HEAL19, CURE2, or $\operatorname{Lr} 271$

\begin{tabular}{|c|c|c|c|c|c|c|c|}
\hline & Malt & + Paracasei & + HEAL 9 & + HEAL 19 & + CURE $2 \mathrm{I}$ & $+\operatorname{Lr} 271$ & $P$ \\
\hline Asparagine & $62 \pm 7$ & $82 \pm 7$ & $89 \pm 9$ & $82 \pm 9$ & $97 \pm 3^{*}$ & $69 \pm 7$ & 0.001 \\
\hline Proline & $175 \pm 2$ & $159 \pm 8$ & $176 \pm 10$ & $217 \pm 10$ & $269 \pm 33^{* * *}$ & $24 I \pm 24 *$ & 0.032 \\
\hline Glycine & $364 \pm 11$ & $438 \pm 18$ & $502 \pm 18 * * * *$ & $458 \pm 28^{*}$ & $404 \pm 25$ & $442 \pm 29$ & 0.002 \\
\hline Phenylalanine & $54 \pm 3$ & $62 \pm 4$ & $74 \pm 6$ & $74 \pm 5$ & $65 \pm 5$ & $85 \pm 9 *$ & 0.007 \\
\hline Glutamine & $680 \pm 26$ & $684 \pm 28$ & $677 \pm 29$ & $633 \pm 33$ & $635 \pm 28$ & $638 \pm 55$ & 0.739 \\
\hline Ammonia & $392 \pm 35$ & $415 \pm 35$ & $361 \pm 40$ & $413 \pm 13$ & $432 \pm 69$ & $430 \pm 55$ & 0.971 \\
\hline Total & $4,375 \pm 139$ & $4,626 \pm 140$ & $4,856 \pm 163$ & $4,755 \pm 192$ & $4,617 \pm 182$ & $4,728 \pm 249$ & 0.477 \\
\hline
\end{tabular}

Results are given as means \pm SEM, $n=6$ for malt + CURE $2 \mathrm{I}$ and $n=7$ for all other groups. Mean values were significantly different from those for rats fed with malt: $* P<0.05, * * P<0.01, * * * P<0.001$. 


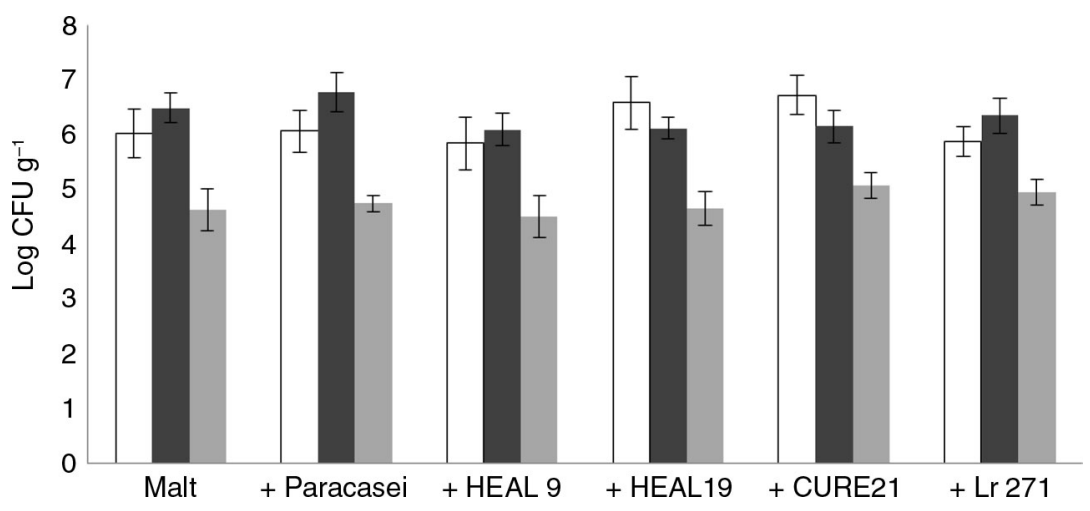

Fig. 1. Viable counts of Bifidobacterium (white bars), Lactobacillus (black bars) and Enterobacteriaceae (grey bars) in the caecum of rats fed malt and malt together with Paracasei, HEAL9, HEAL19, CURE21 or Lr271.

together with $\mathrm{Lr} 271$ could not be repeated either. Instead, there was a decrease in caecal and portal levels of acetic acid when malt was given together with HEAL 9 or Lr 271, and in caecum also with HEAL 19, which might itself be beneficial, since the biosynthesis of cholesterol to a great extent is furnished by acetic acid. Furthermore, there were interesting effects on portal plasma levels of some amino acids proposed to be of metabolic impact; that is, the levels of asparagine, proline, phenylalanine or glycine were higher with all prebiotic strains (HEAL 9, 19, CURE 21, and Lr 271), except Paracasei. However, the levels of glutamine were not affected.

One may wonder why the impact of the administrated probiotics was of such little importance, but could be due to the dose of probiotics given being too low. Another reason would be that the probiotics not reached caecum, which is the actual site for fermentation. However, all probiotic strains, except Cure 21, could be found in the caecum of rats. A more likely explanation would be the physico-chemical characteristics of the fibre in this specific malt, which consisted of a high proportion of insoluble dietary fibre leading to a high unavailability in the hindgut of rats. Differences in physico-chemical properties may also explain why Lr 271 together with

Table 5. Dominant lactobacilli and their incidence in the caecum of rats fed malt and malt with Paracasei, HEAL 9, HEAL 19, CURE 21 or $\operatorname{Lr} 271$

\begin{tabular}{lcccccc}
\hline & & & $+\mathrm{HEAL}$ & $+\mathrm{HEAL}$ & + CURE & $+\mathrm{Lr}$ \\
& Malt & + Paracasei & 9 & 19 & 21 & 271 \\
\hline $\begin{array}{c}\text { Probiotic } \\
\text { strain }\end{array}$ & - & $4 / 7$ & $3 / 7$ & $7 / 7$ & - & $7 / 7$ \\
$\begin{array}{l}\text { L. reuteri } \\
\text { strain }\end{array}$ & $7 / 7$ & $4 / 7$ & $4 / 7$ & $3 / 7$ & $6 / 7$ & $5 / 7$ \\
$\begin{array}{l}\text { L. animalis } \\
\text { strain }\end{array}$ & $6 / 7$ & $3 / 7$ & $7 / 7$ & $0 / 7$ & $5 / 7$ & $4 / 7$ \\
\hline
\end{tabular}

Values are presented as incidence of specific strain per number of test rats. malt increased the levels in caecum and portal blood of rats in a previous study, and not in this study, since that specific malt was suggested to contain high amounts of fibre with low molecular weight, that is, fibres not precipitated in ethanol. Thus, if comparing the fibres in the two studies, the content of dietary fibre polysaccharides (i.e. those precipitated in $80 \%$ ethanol) of malt was considerably higher in this study $(110 \mathrm{~g} / \mathrm{kg}, \mathrm{dwb})$ than in the previous study ( $78 \mathrm{~g} / \mathrm{kg}, \mathrm{dwb})$, whereas the proportion of soluble fibre was lower ( $8 \%$ vs. $30 \%$ in the previous study, if also taking saccharides not precipitated in $80 \%$ ethanol into account) (15). The higher proportion of insoluble fibre resulted in a higher resistance to hindgut fermentation of the fibre in rats. Only $30 \%$ of the dietary fibre was degraded by the hindgut microbiota in this study, while as much as $70 \%$ was degraded in the previous study. It is also preferably oligosaccharides that are referred to as prebiotics, but in principle all fermentable fibres are potential prebiotics, which was not the case for the malt in this study. Anyway these facts show the importance of the substrate in probiotic studies, at least for effects associated to colonic fermentation and the formation of bacterial metabolites.

Decreased caecal levels of SCFAs have been seen in rats administered with probiotics in other studies, and explained by an accelerated absorption into the portal blood (30). However, in this study the portal blood levels of the corresponding SCFAs in caecum were also lower together with probiotics. No difference in the degree of fermentation of the dietary fibre was observed, regardless of whether probiotics were added. This is also consistent with the fact that no differences could be seen in total viable counts of Bifidobacterium, Lactobacillus and Enterobacteriaceae, although the different strains administrated reached caecum. Again, it seems as the chemical structure of the fibre can play a major role, but the exact mechanism behind the reduced formation of SCFAs, resulting from different probiotics in combination with the malt, remains unknown. Interestingly, a change of 
dominant Lactobacilli in caecum could be seen with the strains reducing the formation of SCFAs. It may therefore be speculated, if this could be a reason. However, it cannot be excluded that this is also related to a modified composition of other bacteria in caecum, reflecting the importance to do more in-depth analyses of the microbiota composition. Another reason may be the steric effects and the architecture of the fibre that hinder enzymatic access and bacterial degradation.

None of the probiotic strains had any effects on the glutamine levels in blood. This observation included CURE 21, a probiotic bacterium that has been shown to stimulate the formation of glutamine (21). No effect on caecal SCFA formation could be seen with this bacterium either. This might be due to this probiotic strain not reaching the hindgut of rats, which was also indicated during culturing of dominant Lactobacilli. However, all probiotic strains, except Paracasei, increased plasma portal levels of at least one of the following amino acids, asparagine, glycine, proline and phenylalanine, which is interesting per se. Glycine is an insulin secretagogue and one of the most abundant free amino acids in the blood, and therefore it is interesting that some probiotic strains (HEAL 9 and 19) were able to increase the level of glycine in blood. Albisser et al. (32) showed that glycine could stimulate the excretion of insulin and the glucagon response to a higher extent in diabetic patients than in healthy controls, and Gannon et al. (33) reported a dramatic reduction of the incremental glycaemic area in healthy subjects when glycine was added to a glucose drink. Another interesting amino acid in this respect is phenylalanine, known to be a potent cholecystokinin releaser and so has the capacity to slow down gastric emptying, which may be important for postprandial glucose responses and satiety after a meal (34). In light of this, it is interesting that barley in some studies have been shown to affect carbohydrate metabolism (35).

The increased portal level of amino acids and the absence of effects on caecal SCFA formation indicate that the probiotic strains discussed above had effects already in the small intestine and were thus acting regardless of the dietary fibre. A possible explanation of the enhancement of nutrient absorption with probiotics could be that the probiotic strains facilitate the digestion of peptides in the small intestine, and/or the transportation of amino acids and short peptides from the lumen into the intestinal epithelium. In fact, some studies have shown that probiotics mediated the absorption of nutrients. Breves et al. (36) observed an increased glucose uptake rate in pre-treated pig jejunum tissue with Saccharomyces boulardii and Bacillus cereus var. toyoi, and the co-transport system for dipeptides also tended to increase. Furthermore, in a rat model, the activities of the mucosal enzymes, sucrase and maltase were increased by $S$. boulardii by means of releasing amines (37).

\section{Conclusions}

This study shows that the physico-chemical properties of the dietary fibre are most probably of great importance for the specific SCFAs formed, together with probiotics. In the caecum of rats, the presence of HEAL 9 or $\operatorname{Lr} 271$ in the malt diet decreased the level of acetic acid, which also decreased the levels of acetic acid in the portal blood. Interestingly, a change of dominant Lactobacilli could be seen with these strains. Moreover, some amino acids (glycine, proline, asparagine and phenylalanine) in the portal blood of rats also increased, compared with rats fed malt only. It would be interesting to investigate the mechanism behind this increase in the future.

\section{Acknowledgements}

We would like to thank Dr. Ulf Nilsson and Dr. Greta Jakobsdottir for their technical assistance, Professor Siv Ahrné and Dr. Jie Xu for bacterial assay instruction. We also wish to give thanks to Carlsberg AB (Falkenberg, Sweden) and Probi AB (Lund, Sweden) for kindly supplying the malt and probiotics.

\section{Conflict of interest and funding}

The authors, Y. Zhong and M. Nyman, have no conflict of interests. The study was funded by Albert Påhlsson Foundation, and the author Y. Zhong was financially supported by Chinese Scholarship Council.

\section{References}

1. Galisteo M, Duarte J, Zarzuelo A. Effects of dietary fibers on disturbances clustered in the metabolic syndrome. J Nutr Biochem 2008; 19: 71-84.

2. Pool-Zobel B, Van Loo J, Rowland I, Roberfroid MB. Experimental evidences on the potential of prebiotic fructans to reduce the risk of colon cancer. Brit J Nutr 2002; 87: S273-81.

3. Kvietys PR, Granger DN. Effect of volatile fatty acids on blood flow and oxygen uptake by the dog colon. Gastroenterology 1981; 80: 962-9.

4. Roediger WE. Utilization of nutrients by isolated epithelial cells of the rat colon. Gastroenterology 1982; 83: 424-9.

5. Sakata T. Stimulatory effect of short-chain fatty acids on epithelial cell proliferation in the rat intestine: a possible explanation for trophic effects of fermentable fibre, gut microbes and luminal trophic factors. Br J Nutr 1987; 58: 95-103.

6. Hamer HM, Jonkers D, Venema K, Vanhoutvin S, Troost FJ, Brummer RJ. Review article: the role of butyrate on colonic function. Aliment Pharmacol Ther 2008; 27: 104-19.

7. Meijer K, de Vos P, Priebe M. Butyrate and other short-chain fatty acids as modulators of immunity: what relevance for health? Curr Opin Clin Nutr Metab Care 2010; 13: 715-21.

8. Demigne C, Morand C, Levrat MA, Besson C, Moundras C, Remesy C. Effect of propionate on fatty acid and cholesterol synthesis and on acetate metabolism in isolated rat hepatocytes. Br J Nutr 1995; 74: 209-19.

9. Nilsson A, Ostman E, Preston T, Bjorck I. Effects of GI vs content of cereal fibre of the evening meal on glucose tolerance at a subsequent standardized breakfast. Eur J Clin Nutr 2006; 62: $712-20$. 
10. Nilsson U, Nyman M. Short-chain fatty acid formation in the hindgut of rats fed oligosaccharides varying in monomeric composition, degree of polymerisation and solubility. Br J Nutr 2005; 94: 705-13.

11. Roland N, Nugon-Baudon L, Andrieux C, Szylit O. Comparative study of the fermentative characteristics of inulin and different types of fibre in rats inoculated with a human whole faecal flora. Br J Nutr 1995; 74: 239-49.

12. Berggren A, Björck I, Margareta E, Eggum B. Short-chain fatty acid content and $\mathrm{pH}$ in caecum of rats given various sources of carbohydrates. J Sci Food Agric 1993; 63: 397-406.

13. Nilsson U, Nyman M, Ahrne S, Sullivan EO, Fitzgerald G. Bifidobacterium lactis Bb-12 and Lactobacillus salivarius UCC500 modify carboxylic acid formation in the hindgut of rats given pectin, inulin, and lactitol. J Nutr 2006; 136: 2175-80.

14. Jamar C, Jardin Pd, Fauconnier M. Cell wall polysaccharides hydrolysis of malting barley (Hordeum vulgare L.): a review. Biotechnol Agron Soc Environ 2011; 15: 301-13.

15. Branning CE, Nyman ME. Malt in combination with Lactobacillus rhamnosus increases concentrations of butyric acid in the distal colon and serum in rats compared with other barley products but decreases viable counts of cecal bifidobacteria. J Nutr 2011; 141: 101-7.

16. Robertson JA, I'Anson KJA, Treimo J, Faulds CB, Brocklehurst TF, Eijsink VGH, et al. Profiling brewers' spent grain for composition and microbial ecology at the site of production. LWT Food Sci Technol 2010; 43: 890-6.

17. Kanauchi O, Agata K. Protein, and dietary fiber-rich new foodstuff from brewer's spent grain increased excretion of feces and jejunum mucosal protein content in rats. Biosci Biotechnol Biochem 1997; 61: 29-33.

18. Hanai H, Kanauchi O, Mitsuyama K, Andoh A, Takeuchi K, Takayuki I, et al. Germinated barley foodstuff prolongs remission in patients with ulcerative colitis. Int $\mathbf{J}$ Mol Med 2004; 13: 643-7.

19. Nyman M, Asp NG, Cummings J, Wiggins H. Fermentation of dietary fibre in the intestinal tract: comparison between man and rat. Br J Nutr 1986; 55: 487-96.

20. Molin G, Ahrné S, Jeppsson B, Probi AB. Compositions comprising Lactobacillus plantarum strains in combination with tannin and new Lactobacillus plantarum strains. US patent US7,507,572. 2009.

21. Molin G, Ahrné S, Jeppsson B, Probi AB. New strains of bifidobacterium having the ability to produce glutamine. US patent US7,947,482. 2011.

22. Hutt P, Shchepetova J, Loivukene K, Kullisaar T, Mikelsaar M. Antagonistic activity of probiotic lactobacilli and bifidobacteria against entero- and uropathogens. J Appl Microbiol 2006; 100: 1324-32.

23. Henningsson AM, Bjorck IM, Nyman EM. Combinations of indigestible carbohydrates affect short-chain fatty acid formation in the hindgut of rats. J Nutr 2002; 132: 3098-104.

24. Björck I, Nyman M, Pedersen B, Siljeström M, Asp N-G, Eggum BO. Formation of enzyme resistant starch during autoclaving of wheat starch: studies in vitro and in vivo. J Cereal Sci 1987; 6: 159-72.
25. Asp NG, Johansson CG, Hallmer H, Siljestrom M. Rapid enzymatic assay of insoluble and soluble dietary fiber. J Agric Food Chem 1983; 31: 476-82.

26. Theander O, Aman P, Westerlund E, Andersson R, Pettersson D. Total dietary fiber determined as neutral sugar residues, uronic acid residues, and Klason lignin (the Uppsala method): collaborative study. J AOAC Int 1995; 78: 1030-44.

27. Zhao G, Nyman M, Jonsson JA. Rapid determination of short-chain fatty acids in colonic contents and faeces of humans and rats by acidified water-extraction and direct-injection gas chromatography. Biomed Chromatogr 2006; 20: 674-82.

28. Zhao G, Liu JF, Nyman M, Jonsson JA. Determination of short-chain fatty acids in serum by hollow fiber supported liquid membrane extraction coupled with gas chromatography. J Chromatogr B Analyt Technol Biomed Life Sci 2007; 846: 202-8.

29. Stenberg M, Marko-Varga G, Oste R. Enantioseparation of D- and L-amino acids by a coupled system consisting of an ion-exchange column and a chiral column and determination of D-aspartic acid and D-glutamic acid in soy products. Food Chem 2002; 79: 507-12.

30. Branning C, Hakansson A, Ahrne S, Jeppsson B, Molin G, Nyman M. Blueberry husks and multi-strain probiotics affect colonic fermentation in rats. Brit J Nutr 2009; 101: 859-70.

31. Axling U, Olsson C, Xu J, Fernandez C, Larsson S, Strom K, et al. Green tea powder and Lactobacillus plantarum affect gut microbiota, lipid metabolism and inflammation in high-fat fed C57BL/6J mice. Nutr Metab (Lond) 2012; 9: 105.

32. Albisser AM, Cheng DC, Yamasaki Y, Marliss EB, Zinman B. Changes in blood amino acids account for the insulin and glucagon responses to mixed meals in dogs. Diabetes Res 1985; 2: $49-55$.

33. Gannon MC, Nuttall JA, Nuttall FQ. The metabolic response to ingested glycine. Am J Clin Nutr 2002; 76: 1302-7.

34. Stephens JR, Woolson RF, Cooke AR. Effects of essential and nonessential amino acids on gastric emptying in the dog. Gastroenterology 1975; 69: 920-7.

35. Nilsson AC, Ostman EM, Granfeldt Y, Bjorck IM. Effect of cereal test breakfasts differing in glycemic index and content of indigestible carbohydrates on daylong glucose tolerance in healthy subjects. Am J Clin Nutr 2008; 87: 645-54.

36. Breves G, Walter C, Burmester M, Schröder B. In vitro studies on the effects of Saccharomyces boulardii and Bacillus cereus var. toyoi on nutrient transport in pig jejunum. J Anim Physiol Anim Nutr 2001; 84: 9-20.

37. Buts JP, De Keyser N, De Raedemaker L. Saccharomyces boulardii enhances rat intestinal enzyme expression by endoluminal release of polyamines. Pediatr Res 1994; 36: 522-7.

\footnotetext{
*Yadong Zhong

Applied Nutrition and Food Chemistry

Department of Food Technology, Engineering and Nutrition

Kemicentrum, Lund University

P.O. Box 124, SE-22I 00 Lund, Sweden

Email: Yadong.Zhong@appliednutrition.Ith.se
} 